\title{
ESTABILIDADE ROBUSTA DE SISTEMAS LINEARES EM TEMPO DISCRETO SUJEITOS A ATRASO NO ESTADO
}

\author{
Maurício Zardo Oliveira* \\ mzardooliveiraeyahoo.com.br
}

\author{
Daniel Ferreira Coutinho* \\ danieldee.pucrs.br
}

* Grupo de Automação e Controle de Sistemas, Programa de Pós-Graduação em Engenharia Elétrica, Pontifícia Universidade Católica do Rio Grande do Sul, Av. Ipiranga 6681, Porto Alegre-RS, 90619-900, Brasil.

\begin{abstract}
This paper addresses the robust stability analysis problem of uncertain discrete-time linear systems subject to state delay, where the system state-space representation is allowed to be a rational function of the uncertain parameters. The stability conditions are obtained from Lyapunov-Krasovskii functionals with polynomial parameter dependence by means of nonlinear decompositions of rational vector functions. The proposed results are divided into delay dependent and delay independent, and they are expressed in terms of linear matrix inequalities (LMIs) constraints.
\end{abstract}

KEYWORDS: State delay, Uncertain Discrete-time Systems, Robustness, LMIs.

\section{RESUMO}

Este artigo aborda o problema de análise de estabilidade robusta de sistemas lineares discretos sujeitos a atraso de transporte e incertezas paramétricas que podem aparecer de forma racional na representação por espaço de estados do sistema. As condições de estabilidade são baseadas em funcionais de Lyapunov-Krasovskii e são obtidas através da aplicação de decomposições não lineares de funções vetoriais racionais. As condições de estabilidade propostas podem ser dependentes e independentes do atraso e são expressas através de desigualdades matriciais lineares (ou LMIs).

Artigo submetido em 27/12/2007

1a. Revisão em 11/04/2008

2a. Revisão em 04/08/08

Aceito sob recomendação do Editor Associado

Prof. José Roberto Castilho Piqueira
PALAVRAS-CHAVE: Atraso de Transporte, Sistemas Lineares Incertos em Tempo Discreto, Robustez, LMIs.

\section{INTRODUÇÃO}

Modelos de sistemas dinâmicos sujeitos a incertezas e atrasos de transporte são amplamente encontrados em diversas áreas de conhecimento, como por exemplo, matemática, engenharia, física, economia e biologia (K. Gu et al., 2003). Recentemente, a análise de estabilidade destes sistemas tem despertado grande interesse na área de pesquisa de sistemas de controle (Lien and Chen, 2003).

Ao realizar o projeto de controle, geralmente é desejável a garantia de estabilidade e desempenho dentro dos parâmetros físicos incertos do sistema (Gahinet et al., 1996). Sabese que, além das incertezas, atrasos de transporte prejudicam o desempenho do sistema, podendo levar a instabilidade do sistema em malha fechada (W.-H. Chen et al., 2003). Conseqüentemente, a não inserção do atraso de transporte na fase de projeto do controlador, pode prejudicar a estabilidade e o desempenho do sistema em malha fechada.

No caso de sistemas em tempo discreto, o problema de estabilidade em sistemas com atraso pode ser facilmente contornado através da inserção de pólos na origem na (matriz) função de transferência do sistema. Entretanto, em muitas situações, esta solução não é adequada ou até mesmo não pode ser aplicada. Por exemplo, sistemas na qual o atraso de transporte é várias vezes superior a taxa de amostragem, a inserção de pólos na origem resulta em um sistema de ordem elevada, o que dificulta a utilização de técnicas de otimização convexa na solução de problemas de controle. 
Além disso, quando o atraso não é perfeitamente conhecido ou quando a incerteza é variante no tempo, existe uma dificuldade em representar um sistema equivalente sem o atraso (Suh et al., 2004).

Quando o atraso de transporte não pode ser considerado através da elevação da inserção de integradores discretos, em geral, utilizam-se condições suficientes para provar a estabilidade de sistemas sujeitos a atrasos de transporte de maneira similar ao caso contínuo (K. Gu et al., 2003). Basicamente, existem dois tipos de condições suficientes para analisar a estabilidade: condições que são dependentes e independentes do atraso de transporte (W.-H. Chen et al., 2003). A condição independente do atraso verifica estabilidade do sistema para qualquer valor do atraso de transporte, enquanto que a condição dependente do atraso é menos conservadora, pois o sistema é estável desde que o atraso de transporte não ultrapasse um determinado valor. Tais condições são baseadas em diferentes modelos e transformações, e o sistema é geralmente descrito em termos de equações a diferenças funcionais levando aos conceitos de estabilidade de Lyapunov-Krasovskii e Lyapunov-Razumikhin (K. Gu et al., 2003). Nota-se, através de uma análise na recente bibliografia de sistemas com atraso, que a descrição do problema em termos de desigualdades matriciais lineares (ou LMIs, do inglês Linear Matrix Inequalities (Boyd et al., 1994)) tem sido uma das abordagens mais empregadas para a obtenção de condições de análise e síntese de controle para sistemas com atraso. Por exemplo, os resultados apresentados em (E.Fridman, 2001; Fridman and Shaked, 2003; Lien and Chen, 2003) utilizam uma representação da dinâmica do sistema através de modelos de sistemas descritores (incluindo a classe de sistemas neutrais), e em (Zhou and Li, 2004) são propostas condições de estabilidade independentes do atraso para sistemas descritos por lógica difusa.

Salienta-se que vários esforços têm sido realizados com o objetivo de reduzir o conservadorismo das condições de estabilidade. Em especial, estes esforços têm sido direcionados na busca de técnicas dependentes de parâmetros para determinar a estabilidade de sistemas com incertezas de forma politópica (E.Fridman and Shaked, 2003). Entretanto, grande parte destes resultados foram desenvolvidos para sistemas em tempo contínuo, como por exemplo, as referências (E.Fridman and Shaked, 2003; Suplin et al., 2006). Porém, é conveniente desenvolver condições de estabilidade para sistemas em tempo discreto, visando implementações digitais em tempo real. Apesar do apelo prático, poucos métodos foram desenvolvidos para sistemas discretos, como visto em (W.-H. Chen et al., 2003; Leite and Peres, 2004; Fridman and Shaked, 2003; Xu et al., 2001).

Dentro deste cenário, o presente artigo propõe condições alternativas para estudar a estabilidade robusta de sistemas li- neares incertos em tempo discreto sujeitos a atrasos de transporte. As condições propostas são expressas em termos de restrições LMIs obtidas a partir de funcionais de LyapunovKrasovskii com dependência polinomial no vetor de parâmetros incertos do sistema. Nas próximas seções, os resultados são apresentados através da seguinte estrutura. A Seção 2 formula o problema a ser abordado no artigo. Na Seção 3, apresenta-se uma formulação LMI para tratar o problema de análise de estabilidade de sistemas discretos sujeitos a atraso no estado, considerando funcionais de Lyapunov-Krasovskii quadráticos, enquanto, na Seção 4, consideram-se funcionais de Lyapunov-Krasovskii com dependência polinomial no vetor de parâmetros incertos do sistema. A Seção 5 ilustra a aplicação da metodologia proposta através de exemplos numéricos e por fim na Seção 6 são apresentadas as conclusões e perspectivas futuras deste trabalho.

A seguinte notação será utilizada neste artigo. $I_{n}$ representa uma matriz identidade de dimensão $n \times n, 0_{n}$ e $0_{n \times m}$ representam matrizes de zeros com dimensão $n \times n$ e $n \times m$, respectivamente. Para uma matriz real $P, P^{\prime}$ representa a sua transposta, $\operatorname{He}\{P\}:=P+P^{\prime}$, e $P>0(P \geq 0)$ significa que $P$ é uma matriz simétrica positiva definida (positiva semi-definida). Para um conjunto politópico $\Lambda$, a notação $\mathcal{V}(\Lambda)$ representa o conjunto de todos os vértices de $\Lambda$. Para uma seqüencia $\phi_{k}:[-\tau, 0] \rightarrow \mathbb{R}^{n}$, a norma de $\phi_{k}$ é definida como $\left\|\phi_{k}\right\|_{\tau}:=\sup _{-\tau<k<0}\|\phi(k)\|$ e $x_{k}$ representa a seqüencia de valores de $x(k)$ para $k \in[k-\tau, k]$. As dimensões de vetores e matrizes são omitidas sempre que elas podem ser determinadas pelo contexto.

\section{FORMULAÇÃO DO PROBLEMA}

Considere a seguinte classe de sistemas:

$$
\begin{gathered}
x(k+1)=A(\lambda(k)) x(k)+A_{d}(\lambda(k)) x(k-\tau), \\
x(k)=\phi_{k}, \lambda(k)=\tilde{\phi}_{k}, \forall k \in[-\hat{\tau}, 0],
\end{gathered}
$$

onde $x(k), x(k-\tau) \in \mathbb{R}^{n}$ são os vetores de estados sem $\mathrm{e}$ com atraso, respectivamente, $\lambda(k) \in \mathbb{R}^{n_{\lambda}}$ é o vetor de parâmetros incertos, $\tau \leq \mathbb{R}$ é um número inteiro positivo representando o atraso de transporte $\operatorname{com} \tau \in[0, \hat{\tau}]$, e $\phi_{k}$ e $\tilde{\phi}_{k}$ são as seqüencias de valores iniciais de $x(k)$ e $\lambda(k)$ entre $-\tau$ e 0 . Supõe-se que o vetor de parâmetros incertos e sua variação

$$
\delta \lambda(k):=\lambda(k+1)-\lambda(k),
$$

são limitados a uma região politópica $\Lambda$ com vértices conhecidos, i.e. $(\lambda(k), \delta \lambda(k)) \in \Lambda$, onde a notação $\lambda(k) \in \Lambda$ significa que $(\lambda(k), 0) \in \Lambda$. As matrizes $A(\cdot)$ e $A_{d}(\cdot)$ são funções racionais em $\lambda(k)$ com dimensões apropriadas e bem definidas para todo $\lambda(k) \in \Lambda$.

O problema a ser abordado neste artigo é o de determinar condições que asseguram a estabilidade robusta do sistema 
(1) para todo $(\lambda(k), \delta \lambda(k)) \in \Lambda$, considerando condições dependentes e independentes do atraso $\tau$.

Com este objetivo, considere que a classe de sistemas acima definida possa ser representada pelas seguintes equações de diferenças e algébricas (doravante denominada de representação DAR (Coutinho et al., 2007)):

$$
\begin{gathered}
x(k+1)=A_{1}(\lambda) x(k)+A_{2}(\lambda) x(k-\tau)+A_{3}(\lambda) \pi(k) \\
0 \quad=\Omega_{1}(\lambda) x(k)+\Omega_{2}(\lambda) x(k-\tau)+\Omega_{3}(\lambda) \pi(k)
\end{gathered}
$$

onde $\pi \in \mathbb{R}^{m}$ é um vetor auxiliar; e $A_{1} \in \mathbb{R}^{n \times n}, A_{2} \in$ $\mathbb{R}^{n \times n}, A_{3} \in \mathbb{R}^{n \times m}, \Omega_{1} \in \mathbb{R}^{q \times n}, \Omega_{2} \in \mathbb{R}^{q \times n}$ e $\Omega_{3} \in \mathbb{R}^{q \times m}$ são matrizes afins em $\lambda(k)$. Para que a representação acima seja bem definida, assume-se em relação a representação (2) que:

A1 A matriz $\Omega_{3}(\lambda)$ tem posto completo por colunas para todo $\lambda(k) \in \Lambda$.

A representação acima pode modelar toda a classe de funções vetoriais racionais na incerteza, sendo equivalente à representação NFT (nonlinear fractional transformation) proposta em (Tuan et al., 2003), contrastando com a representação politópica com dependência linear nos parâmetros (Palhares et al., 2005; Leite and Peres, 2004). Claramente, a representação politópica é um caso particular de (2) $\operatorname{com} \pi=$ 0 . Analogamente, a representação DAR em (2) torna-se equivalente às representações LFT (linear fractional transformation (Zhou et al., 1996)) e DAR em (Coutinho et al., 2004) quando as matrizes $A_{1}, A_{2}$ e $A_{3}$ são constantes e $\pi$ é unicamente função da incerteza.

A seguir, para ilustrar a representação DAR, apresenta-se o seguinte exemplo.

Exemplo 1 Considere o seguinte sistema escalar:

$$
x(k+1)=\frac{0.5+\lambda^{2}}{1+\lambda^{3}} x(k)+\frac{0.1}{1+\lambda^{2}} x(k-\tau)
$$

Definindo o vetor auxiliar $\pi(k)$ como

$$
\pi(k)=\left[\begin{array}{lllll}
\frac{x(k)}{1+\lambda^{3}} & \frac{\lambda x(k)}{1+\lambda^{3}} & \frac{\lambda^{2} x(k)}{1+\lambda^{3}} & \frac{x(k-\tau)}{1+\lambda^{2}} & \frac{\lambda x(k-\tau)}{1+\lambda^{2}}
\end{array}\right]^{\prime},
$$

obtém-se a representação em (2) com

$$
\begin{gathered}
A_{1}=0, A_{3}=\left[\begin{array}{lrrrr}
0.5 & 0 & 1 & 0.1 & 0
\end{array}\right], A_{2}=0, \\
\Omega_{1}=\left[\begin{array}{l}
1 \\
0 \\
0 \\
0 \\
0
\end{array}\right], \Omega_{3}(\lambda)=\left[\begin{array}{rrrrr}
-1 & 0 & -\lambda & 0 & 0 \\
\lambda & -1 & 0 & 0 & 0 \\
0 & \lambda & -1 & 0 & 0 \\
0 & 0 & 0 & -1 & -\lambda \\
0 & 0 & 0 & \lambda & -1
\end{array}\right],
\end{gathered}
$$

e $\Omega_{2}=\left[\begin{array}{lllll}0 & 0 & 0 & 1 & 0\end{array}\right]^{\prime}$.
A análise de estabilidade do sistema (1) será desenvolvida com base nos funcionais de Lyapunov-Krasoviskii (FLK) (K. $\mathrm{Gu}$ et al., 2003). Na análise de estabilidade de sistemas incertos sem a presença de atraso de transporte, o método de Lyapunov é largamente utilizado. Neste método, busca-se uma função $V(k, x(k))$, denominada de função de Lyapunov, que quantifica o desvio entre os estados e a solução trivial 0 . Para sistemas com a presença de atraso de transporte, a análise de estabilidade é determinada seguindo o mesmo princípio, porém o conceito de estado inicial é substituído por uma seqüencia inicial $x(\theta)$ no intervalo $\theta \in[-\tau, 0]$ representada, neste artigo, por $\phi_{k}$. Logo, para sistemas com atraso, buscase um funcional $V\left(k, x_{k}\right)$ dependente de $\phi_{k}$, medindo assim o desvio entre a seqüencia $x_{k}$ e a solução trivial 0 . Este método é conhecido como Lyapunov-Krasovskii, e $V\left(k, x_{k}\right)$ é um funcional ao invés de uma função. O seguinte Lema caracteriza a estabilidade de sistemas com atraso segundo esta abordagem (K. Gu et al., 2003).

Lema 1 Considere o sistema (1). Se existir um funcional $V\left(k, x_{k}\right)$ contínuo e escalares positivos $\epsilon_{1}, \epsilon_{2}$ e $\epsilon_{3}$ satisfazendo as seguintes condições

$$
\begin{aligned}
& \epsilon_{1}\|\phi(0)\|^{2} \leq V\left(k, \phi_{k}\right) \leq \epsilon_{2}\left\|\phi_{k}\right\|_{\tau}^{2}, \\
& \Delta V\left(k, \phi_{k}\right) \leq-\epsilon_{3}\|\phi(0)\|^{2},
\end{aligned}
$$

para todo $(\lambda, \delta \lambda) \in \Lambda$, onde

$$
\Delta V\left(k, x_{k}\right):=V(k+1, x(k+1))-V(k, x(k)),
$$

então o sistema (1) é assintoticamente estável para todo $\lambda$ pertencente a $\Lambda$.

\section{ESTABILIDADE QUADRÁTICA}

Nesta seção, trata-se do problema de análise de estabilidade considerando funcionais de Lyapunov-Krasovskii cujos termos são formas quadráticas em $x(k)$ e $x(k-\tau)$ e independentes da incerteza $\lambda(k)$. Em primeiro lugar, aborda-se o caso da estabilidade independente do atraso e em seguida o caso dependente do atraso.

\subsection{Estabilidade Independente do Atraso}

Considere que o funcional de Lyapunov-Krasovskii seja definido da seguinte forma:

$$
V\left(x_{k}\right)=V_{1}(x)+V_{2}\left(x_{k}\right),
$$

onde

$$
\begin{aligned}
V_{1}(x) & =x(k)^{\prime} P x(k), \\
V_{2}\left(x_{k}\right) & =\sum_{r=k-\tau}^{k-1} x(r)^{\prime} Q x(r),
\end{aligned}
$$


com $P$ e $Q$ sendo matrizes simétricas a serem determinadas.

A variação de $V\left(x_{k}\right)$ é determinada através das seguintes relações

$$
\begin{aligned}
\Delta V_{1}(k) & =x(k+1)^{\prime} P x(k+1)-x(k)^{\prime} P x(k), \\
\Delta V_{2}(k) & =\sum_{r=k-\tau+1}^{k} x(r)^{\prime} Q x(r) \\
& -\sum_{r=k-\tau}^{k-1} x(r)^{\prime} Q x(r) .
\end{aligned}
$$

Desenvolvendo $\Delta V_{2}(k)$, tem-se:

$$
\Delta V_{2}(k)=x(k)^{\prime} Q x(k)-x(k-\tau)^{\prime} Q x(k-\tau)
$$

Então, a diferença do FLK fica na forma:

$$
\begin{aligned}
\Delta V(k) & =\left[x(k+1)^{\prime} P x(k+1)-x(k)^{\prime} P x(k)\right. \\
& \left.+x(k)^{\prime} Q x(k)-x(k-\tau)^{\prime} Q x(k-\tau)\right],
\end{aligned}
$$

ou ainda

$$
\Delta V(k)=\eta_{1}(k)^{\prime} \Pi_{1}(P, Q) \eta_{1}(k),
$$

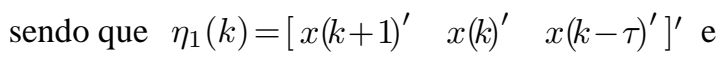

$$
\Pi_{1}(P, Q)=\left[\begin{array}{ccc}
P & 0 & 0 \\
0 & Q-P & 0 \\
0 & 0 & -Q
\end{array}\right]
$$

Note que os elementos $x(k+1), x(k), x(k-\tau)$ do vetor auxiliar $\eta_{1}(k)$ são dependentes entre si, visto que eles estão relacionados pela dinâmica do sistema. Desta forma, considere a seguinte restrição sobre $\eta_{1}(k)$ :

$$
0=\left[\begin{array}{lll}
I_{n} & -A(\lambda) & -A_{d}(\lambda)
\end{array}\right] \eta_{1}(k) .
$$

A restrição acima em termos da representação DAR pode ser reescrita na seguinte forma

$$
0=\Psi_{1}(\lambda) \eta_{1_{a}}(k)
$$

onde $\eta_{1_{a}}(k)=\left[\begin{array}{ll}\eta_{1}(k)^{\prime} & \pi(k)^{\prime}\end{array}\right]^{\prime} \mathrm{e}$

$$
\Psi_{1}(\lambda)=\left[\begin{array}{rrrr}
I_{n} & -A_{1}(\lambda) & -A_{2}(\lambda) & -A_{3}(\lambda) \\
0 & \Omega_{1}(\lambda) & \Omega_{2}(\lambda) & \Omega_{3}(\lambda)
\end{array}\right] .
$$

A partir da formulação acima, propõe-se o seguinte resultado, cujas condições para a análise de estabilidade robusta são independentes do atraso de transporte, onde o FLK é uma forma quadrática em $x$ e $x_{k}$.
Teorema 1 Considere o sistema (1), com a sua representação DAR em (2) satisfazendo A1. Seja $\Lambda$ um politopo com vértices conhecidos, que define os valores admissíveis da incerteza $\lambda(k)$. Suponha que existam matrizes $P>0, Q>0$ e $L$ satisfazendo a seguinte LMI para todo $\lambda \in \mathcal{V}(\Lambda)$.

$$
\left[\begin{array}{cc}
\Pi_{1}(P, Q) & 0 \\
0 & 0_{m}
\end{array}\right]+\operatorname{He}\left\{L \Psi_{1}(\lambda)\right\}<0,
$$

onde as matrizes $\Pi_{1}(P, Q)$ e $\Psi_{1}(\lambda)$ são as definidas em (14) e (17), respectivamente.

Então, o sistema (1) é assintoticamente estável para qualquer $\tau$ e $\lambda \in \Lambda$.

Prova. Suponha que a LMI (18) é satisfeita para todo $\lambda \in$ $\mathcal{V}(\Lambda)$, então por convexidade ela também é satisfeita para todo $\lambda \in \Lambda$.

Como $P$ e $Q$ são definidas positivas, então o funcional definido em (6) satisfaz

$$
V\left(x, x_{k}\right)>0
$$

Além disso, note que $V_{1}$ e $V_{2}$ são formas quadráticas em $x$ e $x_{k}$, respectivamente. Portanto, existem escalares positivos $\epsilon_{1}, \epsilon_{2}$ tais que:

$$
\epsilon_{1}\|x(k)\|^{2} \leq V\left(x, x_{k}\right) \leq \epsilon_{2}\left\|x_{k}\right\|_{\tau}^{2} .
$$

Agora, considere a LMI em (18) e a represente de forma compacta por $\Xi<0$. Como ela é estrita, existe um escalar $\epsilon_{3}$ tal que $\Xi+\epsilon_{3} N_{1}^{\prime} N_{1} \leq 0$, onde $N_{1}$ é uma matriz constante tal que $N_{1} \eta_{1_{a}}(k)=x(k)$, e.g.

$$
N_{1}=\left[\begin{array}{llll}
0_{n} & I_{n} & 0_{n} & 0_{n \times m}
\end{array}\right] .
$$

Pré e pós-multiplicando $\Xi+\epsilon_{3} N_{1}^{\prime} N_{1} \leq 0$ por $\eta_{1_{a}}(k)^{\prime} \mathrm{e}$ $\eta_{1_{a}}(k)$, respectivamente, obtém-se:

$$
\Delta V\left(x, x_{k}\right) \leq-\epsilon_{3}\|x(k)\|^{2}, \forall \lambda \in \Lambda,
$$

visto que $\Psi_{1}(\lambda) \eta_{1_{a}}(k)=0$, e o resto da prova segue a partir do Lema 1.

\subsection{Estabilidade Dependente do Atraso}

Com o objetivo de obter condições de estabilidade dependentes de atraso, considere o seguinte funcional

$$
V\left(x_{k}\right)=V_{1}(x)+V_{2}\left(x_{k}\right)+V_{3}\left(x_{k}\right),
$$

onde $V_{1}$ e $V_{2}$ são como em (7) e (8), respectivamente, e

$$
V_{3}\left(x_{k}\right)=\sum_{\rho=-\tau+1}^{0} \sum_{r=k-1+\rho}^{k-1} y(r)^{\prime} S y(r),
$$


com $S$ sendo uma matriz constante e simétrica a ser determinada e

$$
y(r)=x(r+1)-x(r), \forall r .
$$

De maneira similar a seção anterior, a variação de $V_{3}\left(x_{k}\right)$, i.e. $\Delta V_{3}\left(x_{k}\right)$, é dada por:

$$
\begin{aligned}
\Delta V_{3}\left(x_{k}\right) & =\sum_{\rho=-\tau+1}^{0} \sum_{r=k+\rho}^{k} y(r)^{\prime} S y(r) \\
& -\sum_{\rho=-\tau+1}^{0} \sum_{r=k+\rho-1}^{k-1} y(r)^{\prime} S y(r) \\
& =y(k)^{\prime} \tau S y(k)-\sum_{r=k-\tau}^{k-1} y(r)^{\prime} S y(r) .
\end{aligned}
$$

Portanto, a variação do funcional $V\left(x, x_{k}\right)$ em (22) pode ser escrita da seguinte maneira:

$$
\begin{aligned}
\Delta V\left(x, x_{k}\right) & =\left[x(k+1)^{\prime} P x(k+1)-x(k)^{\prime} P x(k)\right. \\
& +x(k)^{\prime} Q x(k)-x(k-\tau)^{\prime} Q x(k-\tau) \\
& \left.+y(k)^{\prime} \tau S y(k)-\sum_{r=k-\tau}^{k-1} y(r)^{\prime} S y(r)\right] .
\end{aligned}
$$

Por conveniência, todos os termos na expressão acima são colocados dentro do somatório em $r$, ou seja,

$$
\begin{aligned}
\Delta V\left(x, x_{k}\right) & =\frac{1}{\tau} \sum_{r=k-\tau}^{k-1}\left[x(k+1)^{\prime} P x(k+1)-x(k)^{\prime} P x(k)\right. \\
& +x(k)^{\prime} Q x(k)-x(k-\tau)^{\prime} Q x(k-\tau) \\
& \left.+y(k)^{\prime} \tau S y(k)-y(r)^{\prime} \tau S y(r)\right] .
\end{aligned}
$$

De forma compacta, a equação (27) pode ser representada na seguinte maneira:

$$
\Delta V\left(x, x_{k}\right)=\frac{1}{\tau} \sum_{r=k-\tau}^{k-1} \eta_{2}(k, r)^{\prime} \Pi_{2}(P, Q, S, \tau) \eta_{2}(k, r),
$$

onde $\eta_{2}(k, r)=\left[\begin{array}{lllll}x(k+1)^{\prime} & x(k)^{\prime} & x(k-\tau)^{\prime} & y(k)^{\prime} & y(r)^{\prime}\end{array}\right]^{\prime}$ e

$$
\Pi_{2}(P, Q, S, \tau)=\left[\begin{array}{ccccc}
P & 0 & 0 & 0 & 0 \\
0 & Q-P & 0 & 0 & 0 \\
0 & 0 & -Q & 0 & 0 \\
0 & 0 & 0 & \tau S & 0 \\
0 & 0 & 0 & 0 & -\tau S
\end{array}\right] .
$$

Observa-se que os elementos de $\eta_{2}(k, r)$ são dependentes entre si e satisfazem a seguintes restrição:

$$
0=\frac{1}{\tau} \sum_{r=k-\tau}^{k-1}\left[\begin{array}{ccccc}
I_{n} & -A & -A_{d} & 0 & 0 \\
I_{n} & -I_{n} & 0 & -I_{n} & 0 \\
0 & I_{n} & -I_{n} & 0 & -\tau I_{n}
\end{array}\right] \eta_{2}(k, r),
$$

levando em conta a dinâmica do sistema em (1), a definição de $y(\cdot)$ em (24) e

$$
\sum_{r=k-\tau}^{k-1} y(r)=\sum_{r=k-\tau}^{k-1}(x(r+1)-x(r))=x(k)-x(k-\tau) .
$$

Considerando a representação DAR do sistema (1), as restrições acima tomam a seguinte forma

$$
0=\frac{1}{\tau} \sum_{r=k-\tau}^{k-1} \Psi_{2}(\lambda, \tau) \eta_{2_{a}}(k, r),
$$

onde $\eta_{2_{a}}(k, r)=\left[\begin{array}{ll}\eta_{2}(k, r)^{\prime} & \pi(k)^{\prime}\end{array}\right]^{\prime} \mathrm{e}$

$\Psi_{2}(\lambda, \tau)=\left[\begin{array}{cccccc}I_{n} & -A_{1} & -A_{2} & 0 & 0 & -A_{3} \\ 0 & \Omega_{1} & \Omega_{2} & 0 & 0 & \Omega_{3} \\ I_{n} & -I_{n} & 0 & -I_{n} & 0 & 0 \\ 0 & I_{n} & -I_{n} & 0 & -\tau I_{n} & 0\end{array}\right]$.

Com base nos resultados e considerações acima, o seguinte resultado para a análise de estabilidade robusta dependente do atraso é proposto.

Teorema 2 Considere o sistema (1), com a sua representação DAR em (2) satisfazendo A1. Seja $\Lambda$ um politopo com vértices conhecidos, que define os valores admissíveis da incerteza $\lambda(k)$. Seja $\hat{\tau}$ o atraso de transporte máximo. Suponha que existam matrizes $P>0, Q>0, S>0$, e $L$ satisfazendo a seguinte LMI para todo $\lambda \in \mathcal{V}(\Lambda)$.

$$
\left[\begin{array}{cc}
\Pi_{2}(P, Q, S, \hat{\tau}) & 0 \\
0 & 0_{m}
\end{array}\right]+\operatorname{He}\left\{L \Psi_{2}(\lambda, \hat{\tau})\right\}<0,
$$

onde as matrizes $\Pi_{2}(P, Q, S, \hat{\tau})$ e $\Psi_{2}(\lambda, \hat{\tau})$ são as mesmas definidas em (29) e (31), respectivamente.

Então, o sistema (1) é assintoticamente estável para todo $\tau \in$ $[0, \hat{\tau}]$ e todo $\lambda \in \Lambda$.

Prova. Suponha que a LMI (32) é satisfeita para todo $\lambda \epsilon$ $\mathcal{V}(\Lambda)$, então por convexidade ela também é satisfeita para todo $\lambda \in \Lambda$.

Como $P, Q$ e $S$ são definidas positivas, então o funcional definido em (22) satisfaz

$$
V\left(x, x_{k}\right)>0 .
$$


Como $V_{1}$ e $V_{2}, V_{3}$ são formas quadráticas em $x$ e $x_{k}$, respectivamente, existem escalares positivos $\epsilon_{1}, \epsilon_{2}$ tais que:

$$
\epsilon_{1}\|x(k)\|^{2} \leq V\left(x, x_{k}\right) \leq \epsilon_{2}\left\|x_{k}\right\|_{\tau}^{2} .
$$

Agora, considere a LMI em (32) e a represente de forma compacta por $\Xi<0$. Como ela é estrita, existe um escalar $\epsilon_{3}$ tal que $\Xi+\epsilon_{3} N_{2}^{\prime} N_{2} \leq 0$, onde $N_{2}$ é uma matriz constante tal que $N_{2} \eta_{2_{a}}(k)=x(k)$, e.g.

$$
N_{2}=\left[\begin{array}{llllll}
0_{n} & I_{n} & 0_{n} & 0_{n} & 0_{n} & 0_{n \times m}
\end{array}\right] .
$$

Pré e pós-multiplicando $\Xi+\epsilon_{3} N_{2}^{\prime} N_{2} \leq 0$ por $\eta_{2_{a}}(k)^{\prime}$ e $\eta_{2_{a}}(k)$, respectivamente, obtém-se:

$$
\Delta V\left(x, x_{k}\right) \leq-\epsilon_{3}\|x(k)\|^{2}, \forall \lambda \in \Lambda,
$$

visto que $\Psi_{2}(\lambda, \tau) \eta_{2_{a}}(k)=0$, e o resto da prova segue a partir do Lema 1.

\section{ESTABILIDADE DEPENDENTE DE PA- RÂMETROS}

Nesta seção, os resultados apresentados na seção anterior são estendidos para funcionais de Lyapunov-Krasovskii dependentes do vetor de parâmetros $\lambda(k)$. Os termos desses funcionais são formas quadráticas em $x(k)$ e $x(k-\tau)$ e polinomiais em $\lambda(k)$. Em primeiro lugar, aborda-se o caso da estabilidade independente do atraso e em seguida o caso dependente do atraso.

\subsection{Estabilidade Independente do Atraso}

Considere que o funcional de Lyapunov-Krasovskii seja definido da seguinte forma:

$$
V\left(x_{k}, \lambda_{k}\right)=V_{1}(x(k), \lambda(k))+V_{2}\left(x_{k}, \lambda_{k}\right),
$$

onde

$$
\begin{aligned}
V_{1}(x(k), \lambda(k)) & =x(k)^{\prime} \mathcal{P}(\lambda(k)) x(k) e \\
V_{2}\left(x_{k}, \lambda_{k}\right) & =\sum_{r=k-\tau}^{k-1} x(r)^{\prime} \mathcal{Q}(\lambda(r)) x(r),
\end{aligned}
$$

$\mathcal{P}(\lambda)$ e $\mathcal{Q}(\lambda)$ são matrizes simétricas cujos elementos são funções polinomiais em $\lambda$.

Visando uma formulação convexa para o problema de análise de estabilidade robusta, as matrizes $\mathcal{P}(\lambda)$ e $\mathcal{Q}(\lambda)$ obedecem a seguinte estrutura:

$$
\begin{aligned}
& \mathcal{P}(\lambda)=\left[\begin{array}{c}
\Theta(\lambda) \\
I_{n}
\end{array}\right]^{\prime} P\left[\begin{array}{c}
\Theta(\lambda) \\
I_{n}
\end{array}\right], \\
& \mathcal{Q}(\lambda)=\left[\begin{array}{c}
\Theta(\lambda) \\
I_{n}
\end{array}\right]^{\prime} Q\left[\begin{array}{c}
\Theta(\lambda) \\
I_{n}
\end{array}\right],
\end{aligned}
$$

onde $P \in \mathbb{R}^{(n+p) \times(n+p)}$ e $Q \in \mathbb{R}^{(n+p) \times(n+p)}$ são matrizes simétricas e constantes a serem determinadas, e $\Theta(\lambda) \in$ $\mathbb{R}^{p \times n}$ é uma matriz dada cujos elementos são funções polinomiais em $\lambda$.

Definindo o vetor auxiliar $\xi(x, \lambda):=\Theta(\lambda) x$, tem-se que os elementos de $\xi(x, \lambda)$ são funções polinomiais em $\lambda$. Portanto, existe uma representação na forma DAR para $\xi(x, \lambda)$ na seguinte forma (Coutinho et al., 2006):

$$
\begin{gathered}
\xi(x, \lambda)=F_{1}(\lambda) x+F_{2}(\lambda) g(x, \lambda) \\
0=\Phi_{1}(\lambda) x+\Phi_{2}(\lambda) g(x, \lambda)
\end{gathered}
$$

onde $g(x, \lambda) \in \mathbb{R}^{m_{g}}$ é um vetor auxiliar contendo monômios de grau maior do que 2 em $x$ e $\lambda$; e $F_{1} \in \mathbb{R}^{p \times n}$; e $F_{2} \in$ $\mathbb{R}^{p \times m_{g}}, \Phi_{1} \in \mathbb{R}^{q_{g} \times n}$ e $\Phi_{2} \in \mathbb{R}^{q_{g} \times m_{g}}$ são matrizes afins no vetor $\lambda$.

Para que a representação acima seja bem definida, assume-se de maneira similar a representação em (2) que:

A2 A matriz $\Phi_{2}(\lambda)$ tem posto completo por colunas para todo $\lambda(k) \in \Lambda$.

Note que a representação (41) pode ser aplicada para qualquer $x$ e $\lambda$ computados nos instantes $k, k+1$ e $k-\tau$, isto é, para $\xi(k)=\Theta(k) x(k), \xi(k+1)=\Theta(k+1) x(k+1) \mathrm{e}$ $\xi(k-\tau)=\Theta(k-\tau) x(k-\tau)$.

Por conveniência, definem-se os seguintes vetores auxiliares:

$$
v=\left[\begin{array}{l}
\xi(k) \\
x(k)
\end{array}\right], v_{+}=\left[\begin{array}{l}
\xi(k+1) \\
x(k+1)
\end{array}\right], v_{\tau}=\left[\begin{array}{l}
\xi(k-\tau) \\
x(k-\tau)
\end{array}\right] .
$$

Utilizando a notação acima, a diferença do funcional de Lyapunov-Krasovskii como definido em (36) pode ser escrito da seguinte forma:

$$
\Delta V(k)=v_{+}^{\prime} P v_{+}-v^{\prime} P v+v^{\prime} Q v-v_{\tau}^{\prime} Q v_{\tau},
$$

ou na seguinte forma compacta, levando em consideração as representações em (2) e (41),

$$
\Delta V(k)=\eta_{3}(k)^{\prime} \Pi_{3}(P, Q) \eta_{3}(k),
$$

onde $\eta_{3}(k)=\left[\begin{array}{llllllll}v_{+}^{\prime} & v^{\prime} & v_{\tau}^{\prime} & \pi(k)^{\prime} & g_{+}^{\prime} & g^{\prime} & g_{\tau}^{\prime}\end{array}\right]^{\prime} \mathrm{e}$

$$
\Pi_{3}(P, Q)=\left[\begin{array}{cccc}
P & 0 & 0 & 0 \\
0 & Q-P & 0 & 0 \\
0 & 0 & -Q & 0 \\
0 & 0 & 0 & 0_{d_{1}}
\end{array}\right] .
$$

onde $g=g(k), g_{+}=g(k+1), g_{\tau}=g(k-\tau)$ e $d_{1}=m+3 m_{g}$.

Observa-se que os elementos do vetor auxiliar $\eta_{3}(k)$ são dependentes entre si e, com base nas representações DARs em 
(2) e (41), satisfazem as seguintes restrições:

$$
\begin{aligned}
x(k+1)-A_{1}(\lambda) x(k)-A_{2}(\lambda) x(k-\tau)-A_{3}(\lambda) \pi(k) & =0 \\
\Omega_{1}(\lambda) x(k)+\Omega_{2}(\lambda) x(k-\tau)+\Omega_{3}(\lambda) \pi(k) & =0 \\
\xi(k)-F_{1}(\lambda(k)) x(k)-F_{2}(\lambda(k)) g & =0 \\
\xi(k+1)-F_{1}(\lambda+\delta \lambda) x(k+1)-F_{2}(\lambda+\delta \lambda) g_{+} & =0 \\
\xi(k-\tau)-F_{1}(\lambda(k-\tau)) x(k-\tau)-F_{2}(\lambda(k-\tau)) g_{\tau} & =0 \\
\Phi_{1}(\lambda(k)) x(k)+\Phi_{2}(\lambda(k)) g & =0 \\
\Phi_{1}(\lambda(k)+\delta \lambda(k)) x(k+1)+\Phi_{2}(\lambda(k)+\delta \lambda(k)) g_{+} & =0 \\
\Phi_{1}(k-\tau) x(k-\tau)+\Phi_{2}(k-\tau) g_{\tau} & =0
\end{aligned}
$$

as quais podem ser reescritas na seguinte forma compacta

$$
\Psi_{3}(\lambda(k), \delta \lambda(k), \lambda(k-\tau)) \eta_{3}(k)=0
$$

onde $\Psi_{3}(\lambda(k), \delta \lambda(k), \lambda(k-\tau))=\left[\psi_{1(i, j)}\right], i=1, \ldots, 8$ e $j=$ $1, \ldots, 7$, cujos blocos $\psi_{1(i, j)}$ são dados por

$$
\begin{array}{ll}
\psi_{1(1,1)}=N_{x}, & \psi_{1(1,2)}=-A_{1}(\lambda(k)) N_{x}, \\
\psi_{1(1,3)}=-A_{2}(\lambda(k)) N_{x}, & \psi_{1(1,4)}=-A_{3}(\lambda(k)), \\
\psi_{1(2,2)}=\Omega_{1}(\lambda(k)) N_{x}, & \psi_{1(2,3)}=\Omega_{2}(\lambda(k)) N_{x}, \\
\psi_{1(2,4)}=\Omega_{3}(\lambda(k)), & \psi_{1(3,2)}=N_{\xi}-F_{1}(\lambda(k)) N_{x}, \\
\psi_{1(3,6)}=-F_{2}(\lambda(k)), & \psi_{1(4,1)}=N_{\xi}-F_{1}(\lambda+\delta \lambda) N_{x}, \\
\psi_{1(4,5)}=-F_{2}(\lambda+\delta \lambda), & \psi_{1(5,3)}=N_{\xi}-F_{1}(\lambda(k-\tau)) N_{x}, \\
\psi_{1(5,7)}=-F_{2}(\lambda(k-\tau)), & \psi_{1(6,2)}=\Phi_{1}(\lambda(k)) N_{x}, \\
\psi_{1(6,6)}=\Phi_{2}(\lambda(k)), & \psi_{1(7,1)}=\Phi_{1}(\lambda(k+1)) N_{x}, \\
\psi_{1(7,5)}=\Phi_{2}(\lambda(k+1)), & \psi_{1(8,3)}=\Phi_{1}(\lambda(k-\tau)) N_{x}, \\
\psi_{1(8,7)}=\Phi_{2}(\lambda(k-\tau)), &
\end{array}
$$

sendo que as matrizes $\psi_{1(i, j)}$ não definidas acima são matrizes de zeros com dimensões apropriadas, e $N_{\xi}$ e $N_{x}$ são matrizes constantes tais que $N_{\xi} v_{(\cdot)}=\xi(\cdot)$ e $N_{x} v_{(\cdot)}=x(\cdot)$, como, por exemplo, as matrizes

$$
N_{\xi}=\left[\begin{array}{cc}
I_{p} & 0_{p \times n}
\end{array}\right], N_{x}=\left[\begin{array}{ll}
0_{n \times p} & I_{n}
\end{array}\right] .
$$

A formulação acima leva ao seguinte resultado para a análise de estabilidade robusta, independente do atraso de transporte e dependente de parâmetros.

Teorema 3 Considere o sistema (1), com a sua representação DAR em (2) satisfazendo A1. Seja $\Theta(\lambda) \in \mathbb{R}^{p \times n}$ uma dada matriz cujos elementos são funções polinomiais em $\lambda$, e considere a decomposição DAR em (41) satisfazendo A2. Seja $\Lambda$ um politopo com vértices conhecidos, que define os valores admissíveis da incerteza $\lambda(k)$ e de sua variação $\delta \lambda(k)$. Suponha que existam matrizes $P=P^{\prime}$, $Q=Q^{\prime}, L, M$ e $W$ satisfazendo as seguintes LMIs para todo $(\lambda(k), \delta \lambda(k), \lambda(k-\tau)) \in \mathcal{V}(\Lambda \times \Lambda)$.

$$
\begin{aligned}
& {\left[\begin{array}{cc}
P & 0 \\
0 & 0 \\
m_{g}
\end{array}\right]+\operatorname{He}\left\{L \Upsilon_{1}(\lambda(k))\right\}>0} \\
& {\left[\begin{array}{cc}
Q & 0 \\
0 & 0_{m_{g}}
\end{array}\right]+\operatorname{He}\left\{M \Upsilon_{1}(\lambda(k))\right\}>0} \\
& \Pi_{3}(P, Q)+\operatorname{He}\left\{W \Psi_{3}(\lambda(k), \delta \lambda(k), \lambda(k-\tau))\right\}<0
\end{aligned}
$$

onde

$$
\Upsilon_{1}(\lambda)=\left[\begin{array}{cc}
N_{\xi}-F_{1}(\lambda) N_{x} & -F_{2}(\lambda) \\
\Phi_{1}(\lambda) N_{x} & \Phi_{2}(\lambda)
\end{array}\right] .
$$

Então, o sistema (1) é assintoticamente estável para qualquer $(\lambda, \delta \lambda) \in \Lambda$ e independente do atraso $\tau$.

Prova. Suponha que as LMIs (45)-(47) estão satisfeitas para todo $(\lambda(k), \delta \lambda(k), \lambda(k-\tau)) \in \mathcal{V}(\Lambda \times \Lambda)$, então por convexidade elas também estão satisfeitas para todo $(\lambda(k), \delta \lambda(k), \lambda(k-\tau)) \in$ $\Lambda \times \Lambda$.

Considere as LMIs (45) e (46) e defina o vetor auxiliar $\sigma_{1}=$ $\left[\begin{array}{ll}v^{\prime} & g^{\prime}\end{array}\right]^{\prime}$. Pré- e pós-multiplicando estas LMIs por $\sigma_{1}^{\prime} \mathrm{e}$ $\sigma_{1}$, respectivamente, obtém-se:

$$
\begin{gathered}
V_{1}(x(k), \lambda(k))=x(k)^{\prime} \mathcal{P}(\lambda(k)) x(k)>0, \\
V_{2}\left(x_{k}, \lambda_{k}\right)=\sum_{r=k-\tau}^{k-1} x(r)^{\prime} \mathcal{Q}(\lambda(r)) x(r)>0,
\end{gathered}
$$

pois $\Upsilon_{1}(\lambda) \sigma_{1}=0$ para qualquer instante $k$.

Como os elementos das matrizes $\mathcal{P}(\lambda)$ e $\mathcal{Q}(\lambda)$ são limitados por suposição (i.e., $\lambda \in \Lambda$ ) existem escalares positivos $\epsilon_{1}, \epsilon_{2}$ tais que:

$$
\epsilon_{1}\|x(k)\|^{2} \leq V\left(x, x_{k}\right) \leq \epsilon_{2}\left\|x_{k}\right\|_{\tau}^{2} .
$$

Agora, considere a LMI em (47) e a represente de forma compacta por $\tilde{\Xi}<0$. Como ela é estrita, existe um escalar $\epsilon_{3}$ tal que $\tilde{\Xi}+\epsilon_{3} N_{3}^{\prime} N_{3} \leq 0$, onde $N_{3}$ é uma matriz constante tal que $N_{3} \eta_{3}(k)=x(k)$. Pré e pós-multiplicando $\tilde{\Xi}+\epsilon_{3} N_{3}^{\prime} N_{3} \leq 0$ por $\eta_{3}(k)^{\prime}$ e $\eta_{3}(k)$, respectivamente, obtém-se:

$$
\Delta V\left(x, x_{k}\right) \leq-\epsilon_{3}\|x(k)\|^{2}, \forall \lambda \in \Lambda,
$$

visto que $\Psi_{3}(\cdot) \eta_{3}(k)=0$, e o resto da prova segue a partir do Lema 1.

\subsection{Estabilidade Dependente do Atraso}

Afim de obter condições de estabilidade dependente do atraso e do vetor de parâmetros $\lambda$, considere o seguinte FLK

$$
V\left(x_{k}, \lambda_{k}\right)=V_{1}(x(k), \lambda(k))+V_{2}\left(x_{k}, \lambda_{k}\right)+V_{3}\left(x_{k}, \lambda_{k}\right),
$$


onde $V_{1}$ e $V_{2}$ são os mesmos definidos em (37) e (38), respectivamente, $\mathrm{e}$

$$
V_{3}\left(x_{k}, \lambda_{k}\right)=\sum_{\rho=-\tau+1}^{0} \sum_{r=k-1+\rho}^{k-1}\left[\begin{array}{c}
\xi_{y}(r) \\
y(r)
\end{array}\right]^{\prime} S\left[\begin{array}{c}
\xi_{y}(r) \\
y(r)
\end{array}\right],
$$

onde $S \in \mathbb{R}^{(n+p) \times(n+p)}$ é uma matriz simétrica e constante a ser determinada, e

$$
\xi_{y}(r)=\Theta(r+1) x(r+1)-\Theta(r) x(r) .
$$

De maneira similar a seção anterior, definem-se os seguintes vetores auxiliares:

$$
w=\left[\begin{array}{c}
\xi_{y}(k) \\
y(k)
\end{array}\right] \text { e } w_{r}=\left[\begin{array}{c}
\xi_{y}(r) \\
y(r)
\end{array}\right],
$$

levando a seguinte expressão para $\Delta V_{3}\left(x_{k}, \lambda_{k}\right)$ :

$$
\Delta V_{3}\left(x_{k}, \lambda_{k}\right)=w^{\prime} \tau S w-\sum_{r=k-\tau}^{k-1} w_{r}^{\prime} S w_{r} .
$$

Utilizando a notação acima, a diferença do funcional definido em (52) pode ser escrita da seguinte forma:

$$
\begin{array}{r}
\Delta V\left(x_{k}, \lambda_{k}\right)=v_{+}^{\prime} P v_{+}-v^{\prime} P v+v^{\prime} Q v-v_{\tau}^{\prime} Q v_{\tau} \\
+w^{\prime} \tau S w-\sum_{r=k-\tau}^{k-1} w_{r}^{\prime} S w_{r},
\end{array}
$$

Por conveniência, todos os termos na expressão acima são colocados dentro do somatório em $r$, ou seja,

$$
\begin{aligned}
\Delta V\left(x_{k}, \lambda_{k}\right) & =\frac{1}{\tau} \sum_{r=k-\tau}^{k-1}\left[v_{+}^{\prime} P v_{+}-v^{\prime} P v+v^{\prime} Q v\right. \\
& \left.-v_{\tau}^{\prime} Q v_{\tau}+\tau\left(w^{\prime} S w-w_{r}^{\prime} S w_{r}\right)\right] .
\end{aligned}
$$

Levando em consideração (2) e (41), $\Delta V\left(x_{k}, \lambda_{k}\right)$ pode ser representada na seguinte forma compacta

$\Delta V\left(x_{k}, \lambda_{k}\right)=\frac{1}{\tau} \sum_{r=k-\tau}^{k-1} \eta_{4}(k, r)^{\prime} \Pi_{4}(P, Q, S, \tau) \eta_{4}(k, r)$,

onde $\eta_{4}(k, r)=\left[\begin{array}{llllllll}v_{+}^{\prime} & v^{\prime} & v_{\tau}^{\prime} & w & w_{r} & \pi(k)^{\prime} & g_{+}^{\prime}\end{array}\right.$ $\left.g^{\prime} g_{\tau}^{\prime}\right]^{\prime}, \mathrm{e}$

$$
\Pi_{4}(P, Q, S, \tau)=\left[\begin{array}{cccccc}
P & 0 & 0 & 0 & 0 & 0 \\
0 & Q-P & 0 & 0 & 0 & 0 \\
0 & 0 & -Q & 0 & 0 & 0 \\
0 & 0 & 0 & \tau S & 0 & 0 \\
0 & 0 & 0 & 0 & -\tau S & 0 \\
0 & 0 & 0 & 0 & 0 & 0
\end{array}\right]
$$

Note que os elementos do vetor auxiliar $\eta_{4}(k, r)$ são dependentes entre si e considerando a representação DAR (2) do sistema (1) é possível associar as restrições

$$
0=\frac{1}{\tau} \sum_{r=k-\tau}^{k-1} \Gamma(\lambda) \eta_{4}(k, r),
$$

onde

$$
\Gamma(\lambda)=\left[\begin{array}{cccccc}
N_{x} & -A_{1} N_{x} & -A_{2} N_{x} & 0_{n \times d_{2}} & -A_{3} & 0_{n \times d_{3}} \\
0 & \Omega_{1} N_{x} & \Omega_{2} N_{x} & 0_{n \times d_{2}} & \Omega_{3} & 0_{n \times d_{3}}
\end{array}\right]
$$

sendo $d_{2}=2(n+p)$ e $d_{3}=3 m_{g}$.

Com base nas representação DAR definida em (41), obtémse as seguintes igualdades:

$$
\begin{gathered}
\frac{1}{\tau} \sum_{r=k-\tau}^{k-1} \xi(\cdot)-F_{1}(\cdot) x(\cdot)-F_{2}(\cdot) g(\cdot)=0 \\
\frac{1}{\tau} \sum_{r=k-\tau}^{k-1} \Phi_{1}(\cdot) x(\cdot)+\Phi_{2}(\cdot) g(\cdot)=0
\end{gathered}
$$

De maneira similar, levando em conta a definição de $y(\cdot)$ em (24) e definindo os seguintes vetores auxiliares:

$$
v_{r}=\left[\begin{array}{l}
\xi(r) \\
x(r)
\end{array}\right] \text { e } \quad v_{r+}=\left[\begin{array}{l}
\xi(r+1) \\
x(r+1)
\end{array}\right],
$$

então $w=v_{+}-v \mathrm{e}$

$$
\sum_{r=k-\tau}^{k-1} w_{r}=\sum_{r=k-\tau}^{k-1}\left(v_{r+}-v_{r}\right)=v-v_{\tau} .
$$

Considerando a representação DAR do sistema em (2) e de $\xi(\cdot)$ em (41), as restrições acima podem ser reescritas na seguinte forma compacta

$$
0=\frac{1}{\tau} \sum_{r=k-\tau}^{k-1} \Psi_{4}(\lambda(k), \delta \lambda(k), \lambda(k-\tau), \tau) \eta_{4}(k, r)
$$

onde $\Psi_{4}(\lambda(k), \delta \lambda(k), \lambda(k-\tau), \tau)=\left[\psi_{2(i, j)}\right], i=1, \ldots, 10 \mathrm{e}$ $j=1, \ldots, 9$, cujos blocos $\psi_{2(i, j)}$ são dados por

$$
\begin{array}{ll}
\psi_{2(1,1)}=N_{x}, & \psi_{2(1,2)}=-A_{1}(\lambda(k)) N_{x}, \\
\psi_{2(1,3)}=-A_{2}(\lambda(k)) N_{x}, & \psi_{2(1,6)}=-A_{3}(\lambda(k)), \\
\psi_{2(2,2)}=\Omega_{1}(\lambda(k)) N_{x}, & \psi_{2(2,3)}=\Omega_{2}(\lambda(k)) N_{x}, \\
\psi_{2(2,6)}=\Omega_{3}(\lambda(k)), & \psi_{2(3,2)}=N_{\xi}-F_{1}(\lambda(k)) N_{x}, \\
\psi_{2(3,8)}=-F_{2}(\lambda(k)), & \psi_{2(4,1)}=N_{\xi}-F_{1}(\lambda+\delta \lambda) N_{x}, \\
\psi_{2(4,7)}=-F_{2}(\lambda+\delta \lambda), & \psi_{2(5,3)}=N_{\xi}-F_{1}(\lambda(k-\tau)) N_{x}, \\
\psi_{2(5,9)}=-F_{2}(\lambda(k-\tau)), & \psi_{2(6,2)}=\Phi_{1}(\lambda(k)) N_{x}, \\
\psi_{2(6,8)}=\Phi_{2}(\lambda(k)), & \psi_{2(7,1)}=\Phi_{1}(\lambda(k+1)) N_{x}, \\
\psi_{2(7,7)}=\Phi_{2}(\lambda(k+1)), & \psi_{2(8,3)}=\Phi_{1}(\lambda(k-\tau)) N_{x}, \\
\psi_{2(8,9)}=\Phi_{2}(\lambda(k-\tau)), & \psi_{2(9,1)}=I_{n+p}, \\
\psi_{2(9,2)}=-I_{n+p} & \psi_{2(9,4)}=-I_{n+p} \\
\psi_{2(10,2)}=I_{n+p}, & \psi_{2(10,3)}=-I_{n+p}, \\
\psi_{2(10,5)}=-\tau I_{n+p}, &
\end{array}
$$


e, similarmente a seção anterior, as matrizes $\psi_{2(i, j)}$ não definidas acima são matrizes de zeros com dimensões apropriadas.

Com base na formulação acima o seguinte resultado para para a análise de estabilidade robusta, dependente do atraso de transporte e dependente de parâmetros é proposto.

Teorema 4 Considere o sistema (1), com a sua decomposição DAR em (2) satisfazendo A1. Seja $\Theta(\lambda) \in \mathbb{R}^{p \times n}$ uma dada matriz cujos elementos são funções polinomiais em $\lambda$ e considere a representação DAR em (41) satisfazendo A2. Seja $\hat{\tau}$ o atraso de transporte máximo e $\Lambda$ um politopo com vértices conhecidos, que define os valores admissíveis da incerteza $\lambda(k)$ e de sua variação $\delta \lambda(k)$. Suponha que existam matrizes $P=P^{\prime}, Q=Q^{\prime}, S=S^{\prime}, L, M, T$ e $W$ satisfazendo as seguintes LMIs para todo $(\lambda(k), \delta \lambda(k), \lambda(k-\tau)) \in$ $\mathcal{V}(\Lambda \times \Lambda)$.

$$
\begin{aligned}
& {\left[\begin{array}{cc}
P & 0 \\
0 & 0_{m_{g}}
\end{array}\right]+\operatorname{He}\left\{L \Upsilon_{1}(\lambda(k))\right\}>0} \\
& {\left[\begin{array}{cc}
Q & 0 \\
0 & 0_{m_{g}}
\end{array}\right]+\operatorname{He}\left\{M \Upsilon_{1}(\lambda(k))\right\}>0} \\
& {\left[\begin{array}{cc}
S & 0 \\
0 & 0_{d_{4}}
\end{array}\right]+\operatorname{He}\left\{T \Upsilon_{2}(\lambda(k), \delta \lambda(k))\right\}>0} \\
& \Pi_{4}(P, Q, S, \hat{\tau})+ \\
& \quad \operatorname{He}\left\{W \Psi_{4}(\lambda(k), \delta \lambda(k), \lambda(k-\tau), \hat{\tau})\right\}<0
\end{aligned}
$$

onde $d_{4}=2\left(n+p+m_{g}\right)$ e

$$
\Upsilon_{2}(\lambda, \delta \lambda)=\left[\begin{array}{ccc}
I_{n+p} & {\left[\begin{array}{cc}
I_{n+p} & 0
\end{array}\right]} & {\left[\begin{array}{cc}
-I_{n+p} & 0
\end{array}\right]} \\
0 & \Upsilon_{1}(\lambda) & 0 \\
0 & 0 & \Upsilon_{1}(\lambda+\delta \lambda)
\end{array}\right]
$$

Então, o sistema (1) é assintoticamente estável para todo $\tau \in$ $[0, \hat{\tau}]$ e todo $(\lambda, \delta \lambda) \in \Lambda$.

Prova. Suponha que as LMIs (62)-(65) estão satisfeitas para todo $(\lambda(k), \delta \lambda(k), \lambda(k-\tau)) \in \mathcal{V}(\Lambda \times \Lambda)$, então por convexidade elas também estão satisfeitas para todo $(\lambda(k), \delta \lambda(k), \lambda(k-\tau)) \in$ $\Lambda \times \Lambda$.

Utilizando a prova do Teorema 3, as LMIs (62) e (62) implicam que

$$
\begin{gathered}
V_{1}(x(k), \lambda(k))=x(k)^{\prime} \mathcal{P}(\lambda(k)) x(k)>0, \\
V_{2}\left(x_{k}, \lambda_{k}\right)=\sum_{r=k-\tau}^{k-1} x(r)^{\prime} \mathcal{Q}(\lambda(r)) x(r)>0 .
\end{gathered}
$$

Agora, considere a LMI (64) e defina o vetor auxiliar $\sigma_{2}=$ $\left[\begin{array}{lllll}w^{\prime} & v^{\prime} & g^{\prime} & v_{+}^{\prime} & g_{+}^{\prime}\end{array}\right]^{\prime}$. Pré- e pós-multiplicando esta
LMI por $\sigma_{2}^{\prime}$ e $\sigma_{2}$, respectivamente, obtém-se:

$$
V_{3}\left(x_{k}, \lambda_{k}\right)=\sum_{p=-\tau+1}^{0} \sum_{r=k-1+p}^{k-1}\left[\begin{array}{c}
\xi_{y}(r) \\
y(r)
\end{array}\right]^{\prime} S\left[\begin{array}{c}
\xi_{y}(r) \\
y(r)
\end{array}\right]>0,
$$

pois $\Upsilon_{2}(\lambda, \delta \lambda) \sigma_{2}=0$ para qualquer instante $k$.

Como os elementos de $V_{1}, V_{2}$ e $V_{3}$ são limitados por suposição (i.e., $(\lambda, \delta \lambda) \in \Lambda$ ) existem escalares positivos $\epsilon_{1}, \epsilon_{2}$ tais que:

$$
\epsilon_{1}\|x(k)\|^{2} \leq V\left(x, x_{k}\right) \leq \epsilon_{2}\left\|x_{k}\right\|_{\tau}^{2} .
$$

Agora, considere a LMI em (65) e a represente de forma compacta por $\tilde{\Xi}<0$. Como ela é estrita, existe um escalar $\epsilon_{3}$ tal que $\tilde{\Xi}+\epsilon_{3} N_{4}^{\prime} N_{4} \leq 0$, onde $N_{4}$ é uma matriz constante tal que $N_{4} \eta_{4}(k, r)=x(k)$. Pré e pós-multiplicando $\tilde{\Xi}+\epsilon_{3} N_{4}^{\prime} N_{4} \leq 0$ por $\eta_{4}(k, r)^{\prime}$ e $\eta_{4}(k, r)$, respectivamente, obtém-se:

$$
\Delta V\left(x, x_{k}\right) \leq-\epsilon_{3}\|x(k)\|^{2}, \forall(\lambda, \delta \lambda) \in \Lambda,
$$

visto que $\Psi_{4}(\cdot) \eta_{4}(k, r)=0$, e o resto da prova segue a partir do Lema 1.

\section{EXEMPLOS NUMÉRICOS}

A seguir são apresentados dois exemplos numéricos com o objetivo de ilustrar a abordagem proposta.

Exemplo 1: Considere o Exemplo 1 proposto em (Xu et al., 2001), onde conclui-se que o sistema incerto é quadraticamente estável para $\tau=2$. Uma representação politópica para este sistema, de acordo com a representação apresentada em (1), pode ser dada na seguinte forma:

$$
\begin{aligned}
A(\lambda(k)) & =A \pm \alpha \Delta A e \\
A_{d}(\lambda(k)) & =A_{d} \pm \alpha \Delta A_{d},
\end{aligned}
$$

onde

$$
\begin{gathered}
A=\left[\begin{array}{rr}
-0.5 & -0.4 \\
0.2 & -0.6
\end{array}\right], \quad A_{d}=\left[\begin{array}{rr}
0.3 & 0.1 \\
-0.1 & 0.1
\end{array}\right], \\
\Delta A=\left[\begin{array}{ll}
0.045 & 0.03 \\
0.015 & 0.01
\end{array}\right], \quad \Delta A_{d}=\left[\begin{array}{ll}
0.06 & 0.03 \\
0.02 & 0.01
\end{array}\right],
\end{gathered}
$$

e $\alpha$ é um dado escalar tal que o sistema é quadraticamente estável.

Para o sistema acima, aplica-se o resultado proposto no Teorema 1 e verifica-se que o sistema é assintoticamente estável independente do atraso $\tau$ para todo $\alpha \in[0,5.1)$. Em contrapartida, o resultado apresentado em (Xu et al., 2001) prova que o sistema é assintoticamente estável para $\tau=2$ e $\alpha=1$. Agora, supondo que o sistema seja invariante no 
tempo e levando em consideração que as matrizes $P$ e $Q$ no Teorema 1 aparecem de forma isolada, pode-se fazer as matrizes $P$ e $Q$ dependentes dos vértices do politopo sem perda de convexidade. Neste caso, verifica-se que o sistema é assintoticamente estável independentemente do atraso para todo $\alpha \in[0,5.8)$.

Exemplo 2: Considere o exemplo proposto em (Fridman and Shaked, 2003). A este sistema inclui-se uma incerteza racional nos estados levando a seguinte representação:

$$
x(k+1)=\left[\begin{array}{cc}
0.8 & 0 \\
0 & 0.97 \frac{(0.8+\lambda)}{(1-\lambda)}
\end{array}\right] x(k)+\left[\begin{array}{rr}
-0.1 & 0 \\
-0.1 & -0.1
\end{array}\right] x(k-\tau)
$$

onde $\lambda \in[0,0.1]$.

Definindo-se $\pi=\left[\frac{x_{2}}{1-\lambda}\right]$, a representação DAR em (2) é definida com:

$$
\begin{aligned}
& A_{1}=\left[\begin{array}{cc}
0.8 & 0 \\
0 & 0
\end{array}\right], \quad A_{2}=A_{d}, \quad A_{3}=\left[\begin{array}{c}
0 \\
0.97(0.8+\lambda)
\end{array}\right], \\
& \Omega_{1}=\left[\begin{array}{ll}
0 & 1
\end{array}\right], \quad \Omega_{2}=\left[\begin{array}{ll}
0 & 0
\end{array}\right], \quad \text { e } \Omega_{3}=[-1+\lambda] .
\end{aligned}
$$

Aplicando-se o Teorema 2 verifica-se que o o sistema é assintoticamente estável para um atraso de transporte máximo $\hat{\tau}=16$ para qualquer $\lambda \in[0,0.1]$. Ressalta-se que pelo método apresentado em (Fridman and Shaked, 2003) encontrase o mesmo atraso máximo $\hat{\tau} \operatorname{com} \lambda=0.1$, porém neste método não são admitidas incertezas racionais no modelo.

\section{CONCLUSÕES}

Este artigo aborda o problema de estabilidade de sistemas lineares incertos em tempo discreto sujeitos a atraso no vetor de estados, onde admite-se que o sistema possa depender de forma racional do vetor de parâmetros incertos do sistema. Utilizando funcionais de Lyapunov-Krasovskii com dependência polinomial nos parâmetros, obtém-se condições de estabilidade dependente e independentes do atraso em termos de desigualdades matriciais lineares. Destaca-se que a metodologia proposta não utiliza nenhuma transformação para sistemas com atraso distribuído e nem limitantes superiores do produto cruzado de dois vetores a fim de obter condições convexas. Os resultados numéricos demonstram a aplicabilidade da metodologia proposta na análise de estabilidade de sistemas discretos lineares incertos sujeitos a atraso de transporte.

\section{AGRADECIMENTOS}

Os autores agradecem a Coordenação de Aperfeiçoamento de Pessoal de Nível Superior - CAPES e ao Conselho Naci- onal de Desenvolvimento Científico e Tecnológico - CNPq, pelo apoio financeiro, durante a realização deste trabalho.

\section{REFERÊNCIAS}

Boyd, S., Ghaoui, L. E., Feron, E. and Balakrishnan, V. (1994). Linear Matrix Inequalities in Systems and Control Theory, SIAM books.

Coutinho, D., de Souza, C. and Barbosa, K. (2007). Improved Robust $\mathrm{H}_{\infty}$ Filter Design for Discrete-Time Linear Parameter Varying Systems, Preprints of the 3rd IFAC Symposium on System, Structure and Control, Iguassu Falls, Brazil.

Coutinho, D., de Souza, C. and Trofino, A. (2006). Regional Stability Analysis of Implicit Polynomial Systems, Proceedings of the 45th IEEE Conference on Decision and Control, San Diego, CA, pp. 4224-4229.

Coutinho, D. F., Bazanella, A. S., Trofino, A. and Silva, A. S. (2004). Stability Analysis and Control of a Class of Differential-Algebraic Nonlinear Systems, Int. J. Robust and Nonlinear Control 14: 1301-1326.

E.Fridman (2001). New Lyapunov-Krasovskii functionals for stability of linear retarded and neutral type systems, Systems \& Control Letts. 41: 309-319.

E.Fridman and Shaked, U. (2003). Parameter Dependent Stability and Stabilization of Uncertain Time-Delay Systems, IEEE Trans. Automat. Contr. 48(5): 861-866.

Fridman, E. and Shaked, U. (2003). An LMI approach to stability of discrete delay systems, Proc. Eur. Contr. Conf. (ECC'03), Cambridge, UK, pp. 1-6.

Gahinet, P., Apkarian, P. and Chilali, M. (1996). Affine Parameter-Dependent Lyapunov Functions and Real Parametric Uncertainty, IEEE Trans. Automat. Contr. 41(3): 436-442.

K. Gu, V.L. Kharitonov and J. Chen (2003). Stability of TimeDelay Systems, Birkhauser.

Leite, V. and Peres, P. (2004). A convex approach for robust state feedback control of discrete-time systems with state delay, Proc. American Control Conference, Boston, Ma, pp. 2870-2875.

Lien, C. and Chen, J. (2003). Discrete-Delay-Independent and Discrete-Delay-Dependent Criteria for a Class of Neutral Systems, Journal of Dynamic Systems, Measurement and Control 125: 33-41.

Palhares, R., Campos, C., Ekel, P., Leles, M. and D’Angelo, M. (2005). Delay-dependent robust $\mathrm{H}_{\infty}$ control of uncertain linear systems with lumped delays, IEE Proc. Control Theory Appl. 152(1): 27-33. 
Suh, Y. S., Ro, Y. S., Kang, H. J. and Lee, H. H. (2004). Necessary and Sufficient Stability Condition of Discrete State Delay Systems, International Journal of Control, Automation and Systems 2(4): 501-508.

Suplin, V., E.Fridman and Shaked, U. (2006). $\mathrm{H}_{\infty}$ Control of Linear Uncertain Time-Delay Systems-A Projection Approach, IEEE Trans. Automat. Contr. 51(4): 680685.

Tuan, H., Apkarian, P. and Nguyen, T. (2003). Robust Filtering for Uncertain Nonlinearly Parameterized Plants, IEEE Trans. Signal Processing 51(7): 1806-1815.

W.-H. Chen, Z.-H. Guan and X. Lu (2003). Delay-dependent guaranteed cost control for uncertain discrete-time systems with delay, IEE Proc. Control Theory Appl. 150(4): 412-416.

Xu, S., J.Lam and C.Yang (2001). Quadratic Stability and Stabilization of Uncertain Linear Discrete-Time Systems With State Delay, Systems \& Control Letts. 43: $77-84$.

Zhou, K., Doyle, J. and Glover, K. (1996). Robust and Optimal Control, Prentice Hall.

Zhou, S. and Li, T. (2004). Robust Stabilization for Delayed Discrete-Time Fuzzy Systems Via Basis-Dependent Lyapunov-Krasovskii Function, Fuzzy Sets and Systems 151: 139-153. 